\title{
PROSPECTIVE RANDOMIZED STUDY BETWEEN ARTHROCENTESIS AND OPERATIVE ARTHROSCOPY IN THE MANAGEMENT OF TEMPOROMANDIBULAR JOINT INTERNAL DERANGEMENT
}

\author{
Aly M. Atteya ${ }^{1 *} B D S$, Mohamed H. Warda² $P h D$, Mohamed M. Fata ${ }^{3}$ PhD, Ahmad M. Medra ${ }^{4}$ \\ $P h D$, Florencio G. Monje ${ }^{5} P h D$ \\ ABSTRACT
}

INTRODUCTION: Temporomandibular disorders (TMDs) are common conditions and internal derangement is the most common among them. Different methods have been suggested for treatment , beginning with conservative approaches ending with surgery. Nowadays, arthrocentesis and arthroscopy have decreased the need of the more complex surgical procedures. Despite such advancements, there is deficiency in the literature regarding prospective, randomized, clinical studies supporting either both of them. In doing the necessary studies, and comparing the results, it will be essential to develop a standardized criteria for patient selection and treatment options that can be used by all investigators.

OBJECTIVES: To compare between arthrocentesis and operative arthroscopy in the management of patients with internal derangement of temporomandibular joint stage II and III Wilkes.

MATERIALS AND METHODS: a prospective study was done on 40 patients with temporomandibular joint internal derangement and were divided into 2 groups, 20 patients were treated with arthrocentesis and 20 patients were treated with operative arthroscopy.

RESULTS: The pain score was significantly lower in operative arthroscopy. The mouth opening was significantly higher in the operative arthroscopy.

CONCLUSIONS: Operative arthroscopy is better than arthrocentesis as regards the postoperative pain and mouth opening.

KEYWORDS: TMJ, TMJ arthroscopy, arthroscopy, arthrocentesis, internal derangement.

1-Assistant Lecturer of Maxillofacial and Plastic Surgery Department, Faculty of Dentistry, Alexandria University, Alexandria, Egypt.

2-Professor of Maxillofacial and Plastic Surgery, Faculty of Dentistry, Alexandria University, Alexandria, Egypt.

3- Professor of Maxillofacial and Plastic Surgery, Faculty of Dentistry, Alexandria University, Alexandria, Egypt.

4- Professor of Maxillofacial and Plastic Surgery, University Hospital Infanta Cristina, University of Extremadura.

*Corresponding author:

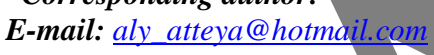

\section{INTRODUCTION}

The temporomandibular joint (TMJ) ginglymo-arthrodial joint and is formed by bony articulations between the mandibular condyle and the glenoid fossa of the temporal bone. The TMJ disc which is a piece of dense avascular fibrous connective tissue, is sited between the condyle and the fossa and splits the joint into two compartments, superior and inferior ones” (1).

TMJ disorders are relatively common conditions with incidence rate of $28 \%$ - $88 \%$. They affect up to one-third of all adults at some stage in their life (2).

Two fundamental components form the temporomandibular system, the temporomandibular joint (TMJ) and the accompanied neuromuscular system. Any defect of one or both components lead to temporomandibular disorder (TMD). Symptoms can be unilateral or bilateral involving the face, head or jaw. TMDs are broadly divided by "the American Academy of Orofacial Pain (AAOP)" into muscle related disorders (myogenous), and joint-related disorders (arthrogenous). The two types can be present concurrently, making diagnosis and treatment more challenging (3).

Internal derangement of the TMJ is one of the most frequent temporomandibular disorders. It was defined by Dolwick (4) in 1983 as "an abnormal relation among the temporomandibular disc in respect to the temporal fossa, the mandibular condyle, and the temporal eminence of the TMJ” (4). It may be present with anterior disc displacement that can be with or without reduction, sometimes with perforation of the articular disc or even the retrodiscal tissue. In late stage, degenerative changes of the joint surfaces may be found. Clinically, it is usually associated with clicking, pain, decrease in the jaw opening, and occasionally locking (5).

In 1989, Wilkes (6) first established a classification which consists of 5 stages depending on the clinical, radiologic, and intraoperative findings.

Many methods have been suggested to treat this entity, starting with conservative approaches. The most frequent measures used among the conservative methods are, medical treatment depending on nonsteroidal antiinflammatory drugs (NSAIDs) and muscle relaxants, Occlusal splint therapy, and physiotherapy. Usually these are the cases with Wilkes stage I that responds well for conservative treatement. Cases that were refractory and showed no actual improvement regarding mandibular function and pain are amenable to more complex options 
such as surgical treatment. Wilkes IV and V patients are usually the ones that will need these more complex procedures (5).

Initially, discectomy was the only surgical option performed, regardless of the type of internal derangement (7). However, in 1979, surgical repositioning of the disc that was known as discoplasty was introduced by Farrar and McCarty (8), finding that it was unnecessary to remove the disc in most of the cases (8).

Later, a small group of surgeons was trying to perform arthroscopic surgery at that time when the majority of surgeons was performing open surgical techniques. In 1975 Ohnishi (9) was the first to introduce this modality which was an evolution in the management of these cases and opened a new phase in the diagnosis and treatment of these conditions (9).

Initially, arthroscopic treatment was mainly executed as lavage of the joint and only later more advanced intra-articular surgical procedures such as lateral capsular release, disc repositioning and disc fixation were added (10).

As a result of the success of arthroscopic lysis and lavage, It has become evident that position of the disc inside the joint is less important than joint mobility. Patients shows good function of the temporomandibular joint with a disc that is anteriorly displaced and non-reducible. This is due to the adaptation of the retrodiscal tissue which act as a pseudodisc (11). It was the recognition of these two concepts that led to the debut of arthrocentesis by Murakami and colleagues (12) in 1987 This technique was further modified by Nitzan et al, (13) in 1991. Since that time, arthrocentesis has been considered as the initial step in the management of most of the cases with TMJ internal derangement and has largely replaced arthroscopic lysis of adhesions and joint lavage (10).

Nitzan et al. have drawn attention to the fact that the displaced disc may not be entirely responsible for acute TMJ closed lock. This idea is based on the excellent results of arthroscopic lysis and lavage. It was held that the success of these procedures was not accompanied by any kind of change in the position or morphology of the disc. Consequently, it was speculated and then demonstrated to think that simply washing the upper articular space accounted for the success of arthroscopic surgery, rather than repositioning the disc. This cast a doubt on the idea of a displaced disc blocking condylar translation in all cases of acute lock (14).

Nowadays, many of the more sophisticated surgical procedures used for the treatment of TMJ internal derangement have been replaced by arthroscopy and arthrocentesis. In spite of this great advancements and their consideration as a minimally invasive procedure, however, clinicians are still facing obstacles in successful treatment of these patients. A review of the literature reveals a lack of prospective, randomized, clinical studies regarding the use of either operative arthroscopy or arthrocentesis for the management of TMDs. In doing the necessary studies, and allowing for direct comparison of the results, it will be important to develop standardized patient selection criteria and treatment options to be used by all investigators (10).

The aim of the study was to compare between arthrocentesis and operative arthroscopy in the management of patients with internal derangement of temporomandibular joint as regards the mandibular movements and the pain score.

The null hypothesis of the present study assumes that no significant difference will be found between operative arthroscopy and arthrocentesis in treating patients suffering from Wilkes stage II and III internal derangement.

\section{MATERIALS AND METHODS}

\section{Ethical considerations}

This study was performed after receiving the approval of the Research Ethics Committee, Faculty of Dentistry, Alexandria University. Informed consent was obtained from all patients. The registration number of the trial in the clinical trials is NCT04308460.

The study was conducted on 40 patients with temporomandibular joint internal derangement. Patients were admitted, investigated and managed in two departments:

1-Maxillofacial and Plastic Surgery Department, Faculty of Dentistry, Alexandria University, Egypt.

2-Oral and Maxillofacial Surgery Department, University Hospital Infanta Cristina, University of Extremadura, Badajoz, Spain.

The study compared between arthrocentesis and operative arthroscopy as regards the post-operative mouth opening in both groups measuring the maximal interincisal opening in millimeters (15) and the post-operative pain score using visual analogue scale (VAS) (16).

Patient selection: Randomized clinical trial

All patients that were included in this study received conservative treatment for 6 months and their symptoms didn't improve, therefore they were indicated for a more invasive procedure.

The patients were randomized using computer based random allocation technique into two groups:

Group I: Twenty patients were treated by Arthrocentesis Group II: Twenty patients were treated by Operative Arthroscopy

Research subjects

Inclusion criteria

The patients with TMJ internal derangement was divided into 5 stages according to Wilkes Classification. Only patients with stages II and III were included in this study.

Exclusion criteria

1-Medically unfit patients as patients suffering from heart diseases, renal failure, liver disease...etc.

2-Stages I, IV \& V Wilkes (Stage I patients will get benefit from conservative treatment and stage IV and V represent an advanced stages).

3- Patients with TMDs secondary to malocclusion.

4-Psychological instability such as depression, mania, paranoia, anxiety and psychosis.

5-Patients undergoing TMJ surgery before due to different TMJ disorders for other TMJ problems such as open surgery for hypermobile joint, open surgery for previous TMJ tumors.

A full history including medical and dental history was taken from all patients. Full clinical examination: general and TMJ examination, routine laboratory investigations and preoperative MRI was also done to all patients. 


\section{Surgical technique}

A- Arthrocentesis (17)

The procedure was carried out under local anesthesia:

The patient was seated at a 45-degree angle turning the head towards the non-affected side. A line was drawn on the skin from the middle of the tragus to the outer canthus of the eye. Entry points were marked along this canthotragal line. The first was marked $10 \mathrm{~mm}$ from the midtragus and $2 \mathrm{~mm}$ below this horizontal line which corresponds to glenoid fossa, the second point corresponding to the articular eminence was marked $10 \mathrm{~mm}$ in front of the first point and $10 \mathrm{~mm}$ below the line. (Figure 1)

Local anesthesia was applied. A 19-gauge needle was introduced into the superior joint space.

Next, $2-3 \mathrm{ml}$ of Marcaine $0.5 \%$ or lignocaine $2 \%$ was injected to distend the upper joint space and anesthetize the adjacent tissues. Another 19-gauge needle was inserted into the distended superior joint space in the area of the articular eminence, enabling free flow of Ringer's solution through the superior compartment (Figure 2). With a syringe, the solution was injected directly in to the joint. On termination of the procedure sodium hyaluronate was injected into the joint, then the needles were removed. B-Arthroscopic technique (18)

All procedures were performed under general anesthesia. Instrumentation: in all procedures, the optical lens was the $2.7 \mathrm{~mm}$ thirty-degree lens of Stryker Endoscopy, Michigan, U.S.A, or the 2,7mm thirty-degree lens of Dyonics, Smith and Nephew company, Watford, United Kingdom. Other instruments include sleeves, sharp and blunt perforators, adhesion knives, an exploratory probe was used. The coblator II of Smith and Nephew was used as a source of energy inside the joint.

The position of the head of the patient was in supine position then the patient's neck was gently turned to place the head in a lateral position.

A marker was used to draw a line between the tragus and the outer canthus (Holmlund and Hellsing line). On this line, $10 \mathrm{~mm}$ in front of the tragus and $2 \mathrm{~mm}$ under this point was usually the site of the first entry which coincided with the maximum concavity of the glenoid fossa. An intramuscular needle was inserted at this point in the medial direction, aiming of injecting anesthetic solution intraarticular to distend the joint and allow ease insertion of the first puncture. (Figure 3 a)

The needle was removed gently and then a cannula with sharp trocar was inserted, with the help of the assistant pulling the mandible downward and forward. Once the joint space was entered, the sharp trocar was changed to the blunt one to avoid undue damage to the joint surfaces. (Figure $3 \mathrm{~b}$ and figure $4 \mathrm{a}$ )

The arthroscope was then inserted into the cannula. (Figure $4 \mathrm{~b}$ ) Ringer lactate was connected to the cannula holding the arthroscope to irrigate the joint continuously in order to distend the joint and maintain the vision clear. After that, a needle was inserted in front or below the first puncture as a drainage port and then exploration of the joint was done. A second puncture was inserted at the anterior recess through direct arthroscopic visualization using the triangulation technique of McCain (18).

Through the second cannula, the coblator was introduced (Figure 5 a) to release any adhesion and cut the anterior attachment of the disc and the neighboring part of the lateral pterygoid muscle. The incision line was located nearly $2-3 \mathrm{~mm}$ in front of the anterior band of the disc and was done across the whole width from medial to lateral. (Figure $5 \mathrm{~b}$ ). After the release, electrocoagulation of the retrodiscal tissue with the coblator was done.

At the end, the obturator was positioned at the anterior border of the disc which then was pushed backwards. The obturator slid along the surface of the disc and arrived in the posterolateral recess. The retrodiscal tissue was pushed down inferiorly and posteriorly . Immediately after, the second cannula was removed and sodium hyaluronate was injected into the joint through the first cannula after removal of the arthroscope.

\section{Postoperative management}

Antibiotics and nonsteroidal anti-inflammatory drugs were routinely prescribed for 3 days. The softness of the postoperative diet was advised for 2 months and was decreased slowly. Exercises were explained to the patient to improve mouth opening and start 1 week after operation.

\section{Follow up}

Pain assessment using VAS (Visual Analogue Scale).

Improvement or persistence of TMJ clicking sound at mouth opening.

Range of motion including maximal interincisal opening, lateral excursion movement and Protrusive movement

\section{Statistical analysis}

Differences in pain scores between groups at different time intervals were compared using Mann Whitney U test with Bonferroni correction. Differences in pain scores across time was detected using Friedman test. Independent t test was performed to compare the mouth opening (M.O) between the study groups while paired t test was used to detect within group changes. Statistical significance level was set at $\mathrm{p}$ value $\leq 0.05$. Data were analyzed using IBM SPSS software package version 20.0. (Armonk, NY: IBM Corp).

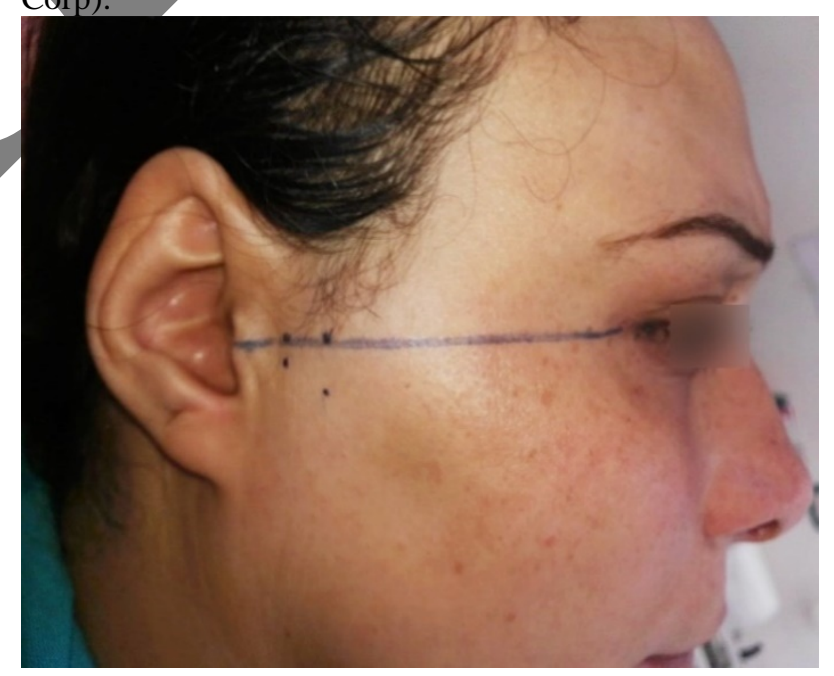

Figure (1): Image shows the cantho-tragal line drawn on the patient, with marking of the two points of entry of the first and second needle. 


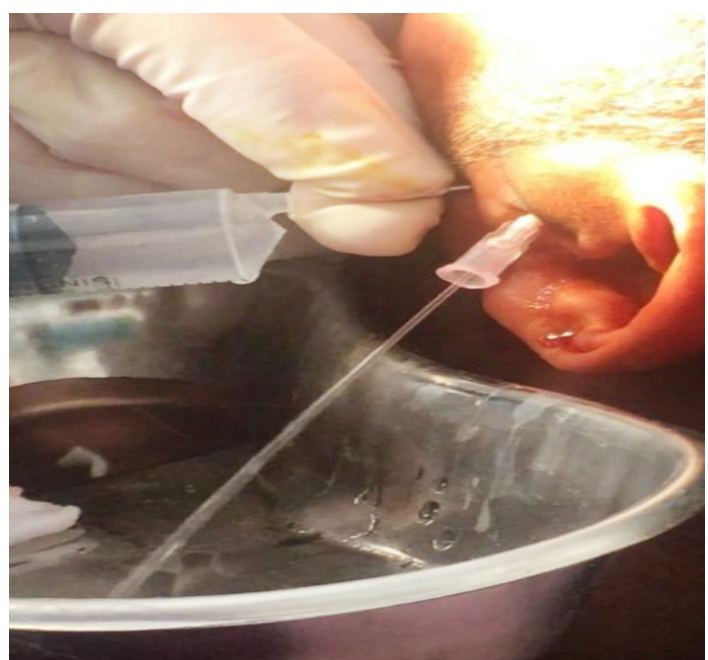

Fig. (2): Image show free flow of Ringer's solution through the superior joint space.

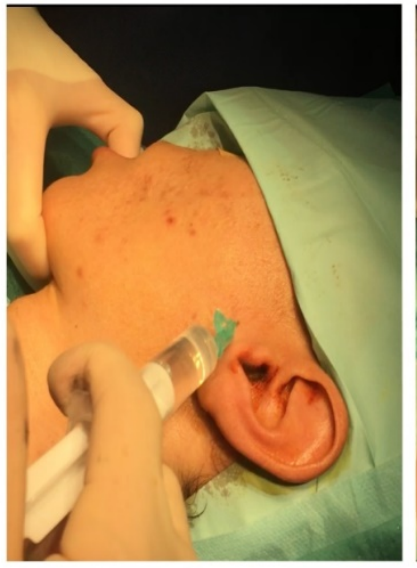

A

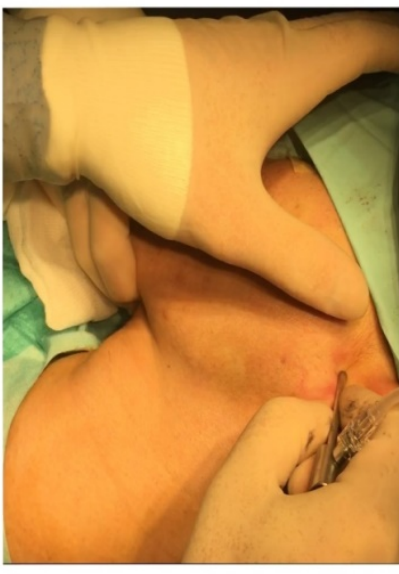

B

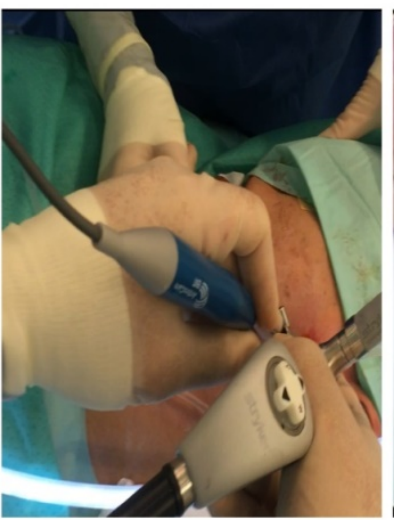

A

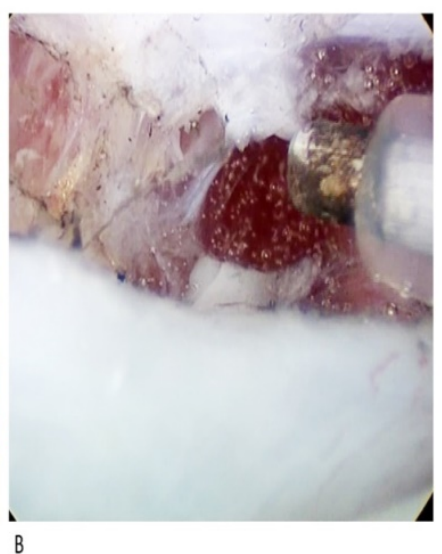

Figure (5): Insertion of the coblator (A) and then view inside the joint doing the lateral pterygoid myotomy (B)

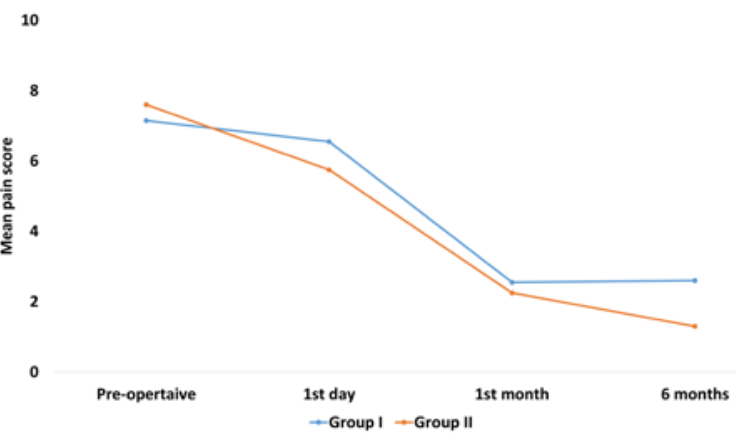

Figure 6: Mean pain score between group I and II at different follow up points

\section{RESULTS}

Forty patients presented to the Maxillofacial and plastic surgery department of the faculty of dentistry, Alexandria University, Egypt and the Oral and Maxillofacial Surgery Department, University Hospital Infanta Cristina, University of Extremadura, Badajoz, Spain with TMJ internal derangement fulfilled the criteria of enrollment in this prospective study.

In the arthrocentesis group (group I) there were 4 males (20\%) and 16 females (80\%) their age ranged from 21 53 years with a mean of $31.95 \pm 8.44$ years. In the operative arthroscopy group (group II), there were only one male (5\%) and 19 females (95\%) their age ranged from 20-62 years with a mean of $37.80 \pm 10.72$ years. There was no statistically significant difference between the 2 groups as regard the sex and the age which demonstrates that single port group is comparable to standard group.

In the arthrocentesis group, there was 12 patients (60\%) with Wilkes stage II and 8 patients with Wilkes stage III (40\%). In the operative arthroscopy group, there was 11 patients with Wilkes stage II (55\%) and 9 patients with Wilkes stage III (45\%). There was statistically insignificant difference between both groups after distribution according to Wilkes stage.

There was not statistically significance regarding the preoperative pain score according to the visual analogue scale between the 2 groups. In the arthrocentesis group it ranged from 5 to 8 with a mean of $7.15 \pm 1.04$. In the operative arthroscopy group, it ranged from 5 to 10 with a mean of $7.60 \pm 1.10$. Regarding the post-operative pain, no statistically 
significance between the 2 groups in the first day nor in the first month. In the arthrocentesis group, the post-operative pain in the first day ranged from 3 to 9 with a mean of $6.55 \pm 1.61$. In the operative arthroscopy group, it ranged from 2 to 9 with a mean of $5.75 \pm 2.07$. After one month, the pain significantly decreases in both groups, it ranged from 1 to 5 with a mean of $2.55 \pm 1.23$ in the arthrocentesis group. In the operative arthroscopy group, it ranged from zero to 6 with a mean of $2.25 \pm 2.40$. When the pain score was compared after 6 months, although both groups had statistically significant decrease of pain, the operative arthroscopy group was statistically significantly lower than the arthrocentesis group, with a pain score ranged from zero to 7 with a mean of $1.30 \pm 2.08$. In the arthrocentesis group it ranged from zero to 6 with a mean of $2.60 \pm 1.54$.

Table (1) demonstrates the comparison between the two studied group according to pain score.

Figure (6) demonstrates Mean pain score between group I and II at different follow up points.

The mouth opening (maximum interincisal opening) preoperative was statistically non-significant between the 2 groups, it ranged from 20 to $34 \mathrm{~mm}$ with a mean of $28.30 \pm 4.94$ $\mathrm{mm}$ in the arthrocentesis group and 22 to $40 \mathrm{~mm}$ with a mean of $31.55 \pm 5.56 \mathrm{~mm}$ in the operative arthroscopy group. In both groups, there was statistically significant increase in the mouth opening after 6 months postoperatively, however, the study got the result that it was statistically significantly higher in the operative arthroscopy group with a mean of $35.40 \pm 5.33 \mathrm{~mm}$. In the arthrocentesis group it had a mean of $31.95 \pm 2.11 \mathrm{~mm}$.

Table (2) demonstrates comparison between the two studied groups according to mouth opening (maximum interincisal opening) after 6 months.

\section{Postoperative follow up:}

All patients were subjected to postoperative follow up regularly, for 6 months to detect any postoperative complications and access the pain score, measures the mandibular movements and detect the absence or presence of clicking. No reported cases of postoperative complications for both groups in this study.

Table (1): Comparison between the two studied groups according to pain score

\begin{tabular}{|c|c|c|c|c|}
\hline \multicolumn{2}{|l|}{ Pain Score } & \multirow{2}{*}{$\begin{array}{l}\begin{array}{l}\text { Group I } \\
(\mathrm{n}=20)\end{array} \\
7.50(6.50- \\
8.00)\end{array}$} & \multirow{2}{*}{$\begin{array}{l}\begin{array}{l}\text { Group II } \\
(\mathrm{n}=20)\end{array} \\
8.0(7.0-8.0)\end{array}$} & \multirow{3}{*}{$\begin{array}{l}P \text { value } \\
0.253\end{array}$} \\
\hline \multirow[t]{2}{*}{$\begin{array}{l}\text { Pre- } \\
\text { operative }\end{array}$} & $\begin{array}{l}\text { Median } \\
\text { (IQR) }\end{array}$ & & & \\
\hline & Min - Max & $5.0-8.0$ & $5.0-10.0$ & \\
\hline \multirow[t]{2}{*}{$1^{\text {st }}$ day } & $\begin{array}{l}\text { Median } \\
\text { (IQR) }\end{array}$ & $7.0(5.5-8.0)$ & $6.0(4.5-7.0)$ & \multirow[t]{2}{*}{0.265} \\
\hline & Min - Max & $3.0-9.0$ & $2.0-9.0$ & \\
\hline \multirow[t]{2}{*}{$1^{\text {st }}$ month } & $\begin{array}{l}\text { Median } \\
\text { (IQR) }\end{array}$ & $2.0(2.0-3.0)$ & $1.0(0.0-5.0)$ & \multirow[t]{2}{*}{0.201} \\
\hline & Min - Max & $1.0-5.0$ & $0.0-6.0$ & \\
\hline \multirow[t]{2}{*}{6 months } & $\begin{array}{l}\text { Median } \\
\text { (IQR) }\end{array}$ & $2.0(2.0-3.50)$ & $0.0(0.0-2.0)$ & \multirow[t]{2}{*}{$0.005^{*}$} \\
\hline & Min - Max & $0.0-6.0$ & $0.0-7.0$ & \\
\hline \multicolumn{2}{|l|}{$P$ value } & $\begin{array}{l}P_{1}=1.00 \\
P_{2}=<0.0001^{*} \\
P_{3}=<0.0001^{*}\end{array}$ & $\begin{array}{l}P_{1}=0.850 \\
P_{2}=<0.0001^{*} \\
P_{3}=<0.0001^{*}\end{array}$ & \\
\hline
\end{tabular}

*Statistically significant at $p$ value $\leq 0.05$

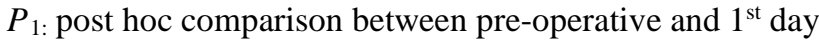
post operatively

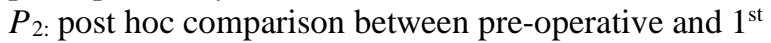
month post operatively
$P_{3:}$ post hoc comparison between pre-operative and 6 months post operatively

Table (2): Comparison between the two studied groups according to M.O

\begin{tabular}{|l|l|l|l|}
\hline \multirow{2}{*}{ M.O (mm) } & $\begin{array}{l}\text { Group I } \\
(\mathbf{n = 2 0})\end{array}$ & $\begin{array}{l}\text { Group II } \\
(\mathbf{n = 2 0})\end{array}$ & \multirow{2}{*}{ P value } \\
\cline { 2 - 3 } & Mean \pm SD & \\
\hline $\begin{array}{l}\text { Pre- } \\
\text { operative }\end{array}$ & $28.30 \pm 4.94$ & $31.55 \pm 5.56$ & 0.058 \\
\hline $\begin{array}{l}\text { Post- } \\
\text { operative }\end{array}$ & $31.95 \pm 2.11$ & $35.40 \pm 5.33$ & $0.013^{*}$ \\
\hline $\boldsymbol{P}$ value & $0.005^{*}$ & $0.001^{*}$ & \\
\hline
\end{tabular}

*Statistically significant at $p$ value $\leq 0.05$

\section{DISCUSSION}

Temporomandibular joint disorders (TMDs) have been classified depending on the cause and the origin of the problem into myogenous, and arthrogenous TMDs (19).Internal derangement is a type of arthrogenous TMDs that usually presents with TMJ clicking sounds and pain, jaw deviation toward the affected side, and decrease of the jaw opening (20).

The reported treatment ways for arthrogenous TMDs involves three sequences. First, conservative treatments which include many options such as medications, occlusal splints and physiotherapy. Second, less invasive treatments which include intraarticular injection of pharmacologic substance such as sodium hyaluronic acid, corticosteroid or platelet-rich plasma. Arthrocentesis or arthroscopic lysis and lavage are also considered less invasive treatments options. Third sequence include surgical treatment such as minimally invasive operative arthroscopic procedures or more invasive open joint surgeries which can be plication of the TMJ disc, discectomy and even arthroplasty. Currently, the best treatment modality with anticipated outcomes based on strong evidence is still not clear (21).

Usually patients suffering from stage I Wilkes will benift from the conservative treatment and will not need more intervention. Patients suffering from stage IV Wilkes (which have osteoarthritis of the condyle) may benefit from minimally invasive surgey but usually from operative arthroscopy more than arthrocentesis, and if they will not improve, usually open surgery is done. Patients suffering from Stage V wilkes (patients have a perforeted disc) usually will need more complex surgical intervention as discectomy and total joint replacement (21). That's why these patients were excluded from this study.

In the present study, there were 4 males (20\%) and 16 females (80\%) in the arthrocentesis group (group I), while in the operative arthroscopy group(group II), there were only one male (5\%) and 19 females (95\%) which indicates that TMDs are more common among females, and this was in agreement with the study of Ahmed $\mathrm{N}$ et al., (22) which had 244 patients , among them there were 31 males (13\%) their age ranged from 17-68 with a mean of 38 years, and 213 females (87\%) their age ranged from 17-68 years with a mean of 35 years.

Regarding the post-operative pain, there is no statistically significance difference between the 2 groups in the first day nor after the first month. In the arthrocentesis 
group, the post-operative pain in the first day ranged from 3 to 9 with a mean of $6.55 \pm 1.61$ while in the operative arthroscopy group it ranged from 2 to 9 with a mean of 5.75 \pm 2.07 . After one month, the pain decreases in both groups, it ranged from 1 to 5 with a mean of $2.55 \pm 1.23$ in the arthrocentesis group, and zero to 6 with a mean of $2.25 \pm 2.40$ in the operative arthroscopy group. The post-operative pain in both groups is due to capsulitis of the joint capsule, subcutaneous tissue edema and inflammation. The pain decreases dramatically after one month in both groups after subsidence of tissue edema and inflammation.

To the best of this study knowledge, no study in the current literature comparing arthrocentesis to arthroscopy reported the post-operative pain score in the first day after the procedure (23).

When the pain score was compared after 6 months, the operative arthroscopy group was statistically significantly lower than the arthrocentesis group, with a pain score ranged from zero to 7 with a mean of $1.30 \pm 2.08$ compared to a pain score ranged from zero to 6 with a mean of $2.60 \pm 1.54$. The significant decrease of pain in the operative arthroscopy group in comparison to the arthrocentesis group is because that in arthrocentesis procedure, lavage only was done which washed away the degrading products that provoke thus significantly reducing the pain. However, in the operative arthroscopy procedure, in addition to the lavage done in the arthrocentesis procedure and its effect, coblation of any synovitis inside the joint and electrocoagulation of the retrodiscal tissue was done and in consequence pain is reduced more than lavage only.

These results are, however, inconsistent with the results of the studies of Murakami et al., (24) Hobeich et al., (25) Fridrich et al., (26) Goudot et al., (27) and Xu et al (28). All these studies found statistically insignificance difference in post-operative pain score between arthrocentesis and arthroscopy. In the study of Murakami et al., (24) the mean post-operative pain was 2.4 and 3.0 in the arthrocentesis group and the operative arthroscopy respectively. Hobeich et al., (25) in their study had the mean post-operative pain 2.55 in the arthrocentesis group and 2.32 in the arthroscopy group. Fridrich et al., (26) showed a mean post-operative pain of 2.3 in the arthrocentesis group, while 1.7 in the arthroscopy group, In the study of Goudot et al., (27) they had a mean postoperative pain of 0.9 in the arthrocentesis group and 1.9 in the arthroscopy group. $\mathrm{Xu}$ et al., (28) found a mean post-operative pain of 0.73 and 1.19 in both the arthrocentesis and the arthroscopy group respectively. The statistically insignificance regarding the post-operative pain score in these studies between arthrocentesis and arthroscopy is because the arthroscopic technique used in these study was arthroscopic lysis and lavage (not operative arthroscopy) which removes the catabolites generated from the inflammatory process and eliminates any intraarticular effusion in the same way that the arthrocentesis did. None of the studies used operative arthroscopy technique which in addition to this, coblation of synovitis and retrodiscal tissue can be done which further reduces pain as mentioned before.

The mouth opening (MIO) after 6 months was statistically significantly higher in the operative arthroscopy group with a mean of $35.40 \pm 5.33 \mathrm{~mm}$ compared to the arthrocentesis group with a mean of $31.95 \pm 2.11 \mathrm{~mm}$. The significant increase of mouth opening in the operative arthroscopy group in comparison to the arthrocentesis group is because that in arthrocentesis lavage of the joint was done trying to break down and lysis any adhesions inside the joint as well as injection of sodium hyaluronate to maintain the normal cartilage surface and protecting the synovial membrane and thus ensuring proper function. In consequence to that improvement of the movement of the disc and significant increase in mouth opening postoperatively compared to preoperatively occurred. On the other hand, lateral pterygoid myotomy was done in the operative arthroscopy, therefore, freeing the disc and allowing better movement of the disc. Added to this, coblation and removal of any adhesions inside the joint and electrocoagulation of retrodiscal tissue was done, the later induce fibrosis in the retrodiscal tissue and in consequence trying to pull the disc backwards to its normal position. That is why significance increase in MIO was noted in the operative arthroscopy group compared to the arthrocentesis group.

These results are consistent with the results of the study of Goudot et al., (27) and Xu et al (28). In the study of Goudot et al., (27) the post-operative mean MIO in the arthrocenetesis group was $33.8 \mathrm{~mm}$ compared to $38.6 \mathrm{~mm}$ in the arthroscopy group. Their study explained this as may be that an active stretching of the muscles is obtained by intubation and intra-operative manipulation. During general anaesthesia and muscular relaxation. Xu et al., (28) got a mean post-operative MIO of $35.7 \mathrm{~mm}$ in the arthrocentesis group and $37.1 \mathrm{~mm}$ in the arthroscopy group. According to their study, arthroscopic lavage has been considered as a more effective procedure because of the larger diameter of portal used in lavage that would enable more extensive removal of inflammatory mediators.

On the other hand, the results of the present study disagreed with the study of Murakami et al., (24) and Fridrich et al., (26) who found that there was no statistically significance difference between arthrocentesis and arthroscopy regarding postoperative MIO. In the study of Murakami et al., (24) the mean postoperative MIO was 42.5 in the arthrocentesis group and 42.1 in the arthroscopy group. According to their study, once arthrocentesis is indicated in the appropriate patients, the clinical efficacy is favorably comparable to the arthroscopic surgery. The mechanism of treatment sequence is probably owed to the lavage of pathological fluid inside the joint followed by the discontinuation of vacuum effect in the locking joint. Fridrich et al., (26) in their study got a mean postoperative MIO of $41 \mathrm{~mm}$ in the arthrocentesis group and $47.5 \mathrm{~mm}$ in the arthroscopy group. According to them, when lysis and lavage, either arthroscopically or by arthrocentesis was done, this will lead to decrease in the inflammation and pain and as a result an increase in the jaw mobility occurred (26).

Arthrocentesis and arthroscopy are not complications free. Generally, the potential complications of arthroscopy can also happen in arthrocentesis, but the incidence and severity of complications are less in arthrocentesis (29). Damage to intraarticular structures by uncareful manipulation or breakage of the instruments, damage of the facial and auriculotemporal nerves, hemorrhage or hematoma due to injury to the superficial temporal vessels, perforation of the external auditory canal and tympanic membrane have been described as complications after 
arthroscopy (30). No complication was found in both the arthrocentesis and the arthroscopy group in this study.

Finally, we should admit that in this study we don't compare apples to apples and that the equivalent to arthrocentesis would be arthroscopic lysis and lavage or primary arthroscopy and not the advanced arthroscopy. In the future we need more data and further studies in that regards.

\section{CONCLUSION}

Both Arthrocentesis and operative arthroscopy are effective methods for the treatment of temporomandibular joint internal derangement, as both had significantly decrease in postoperative pain and increase in mouth opening. However, Operative arthroscopy is significantly better than arthrocentesis regarding the post-operative pain reduction and the increase in mouth opening.

\section{CONFLICT OF INTEREST}

The authors declare that they have no conflicts of interest.

\section{REFERENCES}

1. .Zhang S, Liu X, Yang X, Yang C, Chen M, Haddad MS, et al. Temporomandibular joint disc repositioning using bone anchors: an immediate post surgical evaluation by Magnetic Resonance Imaging. . BMC Musculoskelet Disord 2010;11:262.

2. Greene CS, Marbach JJ. Epidemiologic studies of mandibular dysfunction: a critical review. J Prosthet Dent 1982;48:184-90.

3. Seifeldin SA, Elhayes KA. Soft versus hard occlusal splint therapy in the management of temporomandibular disorders (TMDs). Saudi Dent J 2015;27:208-14.

4. Dolwick MF, Katzberg RW, Helms CA. Internal derangements of the temporomandibular joint: fact or fiction? J Prosthet Dent 1983;49:415-8.

5. Gonzalez-Garcia R, Gil-Diez Usandizaga JL, Rodriguez-Campo FJ. Arthroscopic anatomy and lysis and lavage of the temporomandibular joint. Atlas Oral Maxillofac Surg Clin North Am 2011;19:131-44.

6. Wilkes $\mathrm{CH}$. Internal derangements of the temporomandibular joint. Pathological variations. Arch Otolaryngol Head Neck Surg 1989;115:469-77.

7. Kiehn CL, Desprez JD. Meniscectomy for internal derangement of temporomandibular joint. $\mathrm{Br} \mathrm{J}$ Plast Surg 1962;15:199-204.

8. Farrar WB, McCarty WL Jr. The TMJ dilemma. J Ala Dent Assoc 1979;63:19-26.

9. Onishi M. [Arthroscopy of the temporomandibular joint (author's transl)]. Kokubyo Gakkai Zasshi 1975;42:20713.

10. Laskin DM. Temporomandibular disorders: the past, present, and future. Odontology 2007;95:10-5.

11. Laskin D. Surgical management of internal derangements. Temporomandibular disorders: an evidenced-based approach to diagnosis and treatment Chicago: Quintessence Publishers 2006:469-81.

12. Murakami KI, Iizuka $\mathrm{T}$, Matsuki $\mathrm{M}$, Ono $\mathrm{T}$. Recapturing the persistent anteriorly displaced disk by mandibular manipulation after pumping and hydraulic pressure to the upper joint cavity of the temporomandibular joint. Cranio 1987;5:17-24.
13. Nitzan DW, Dolwick MF, Martinez GA. Temporomandibular joint arthrocentesis: a simplified treatment for severe, limited mouth opening. J Oral Maxillofac Surg 1991;49:1163-7.

14. Monje-Gil F, Nitzan D, Gonzalez-Garcia R. Temporomandibular joint arthrocentesis. Review of the literature. Med Oral Patol Oral Cir Bucal 2012;17: 575-81.

15. Celic R, Jerolimov V, Knezovic Zlataric D, Klaic B. Measurement of mandibular movements in patients with temporomandibular disorders and in asymptomatic subjects. Coll Antropol 2003;27:43-9.

16. Huskisson EC. Measurement of pain. Lancet 1974;2:1127-31.

17. Nitzan DW. Temporomandibular joint arthrocentesis: biologic basis and treatment outcome. In: Stegenga B, de Bont LGM, (eds). Management of Temporomandibular Joint Degenerative Diseases: Biologic Basis and Treatment Outcome. Basel: Birkhäuser Basel; 1996. pp.113-23.

18. McCain JP, de la Rua $H$, LeBlanc WG. Puncture technique and portals of entry for diagnostic and operative arthroscopy of the temporomandibular joint. Arthroscopy 1991;7:221-32.

19. Dworkin SF, LeResche L. Research diagnostic criteria for temporomandibular disorders: review, criteria, examinations and specifications, critique. J Craniomandib Disord 1992;6:301-55.

20. Okeson J. Etiology and identification of functional disturbances in masticatory system. management of temporomandibular joint and occlusion $7^{\text {th }}$ ed: Elsevier; 2013.

21. Al-Moraissi EA, Wolford LM, Ellis E, 3rd, Neff A. The hierarchy of different treatments for arthrogenous temporomandibular disorders: A network meta-analysis of randomized clinical trials. J Craniomaxillofac Surg 2020;48:9-23.

22. Ahmed N, Sidebottom A, O'Connor M, Kerr HL. Prospective outcome assessment of the therapeutic benefits of arthroscopy and arthrocentesis of the temporomandibular joint. Br J Oral Maxillofac Surg 2012;50:745-8.

23. Al-Moraissi EA. Arthroscopy versus arthrocentesis in the management of internal derangement of the temporomandibular joint: a systematic review and metaanalysis. Int J Oral Maxillofac Surg 2015;44:104-12.

24. Murakami K, Hosaka H, Moriya Y, Segami N, Iizuka T. Short-term treatment outcome study for the management of temporomandibular joint closed lock. A comparison of arthrocentesis to nonsurgical therapy and arthroscopic lysis and lavage. Oral Surg Oral Med Oral Pathol Oral Radiol Endod 1995;80:253-7.

25. Hobeich JB SZ, Ismail E, Sadig WM, Hokayem NE, Almas K. Arthroscopy versus arthrocentesis. A retrospective study of disc displacement management without reduction. Saudi Med J 2007;28:1541-4.

26. Fridrich KL, Wise JM, Zeitler DL. Prospective comparison of arthroscopy and arthrocentesis for temporomandibular joint disorders. J Oral Maxillofac Surg 1996;54:816-20.

27. Goudot P, Jaquinet AR, Hugonnet S, Haefliger W, Richter M. Improvement of pain and function after 
Atteya et al.

Operative arthroscopy versus arthrocentesis in TMJ internal derangement.

arthroscopy and arthrocentesis of the temporomandibular joint: a comparative study. J Craniomaxillofac Surg 2000;28:39-43.

28. Xu Y, Lin H, Zhu P, Zhou W, Han Y, Zheng Y, et al. A comparative study between use of arthroscopic lavage and arthrocentesis of temporomandibular joint based on computational fluid dynamics analysis. PLoS One 2013;8:e78953.

29. Tan DBP, Krishnaswamy G. A Retrospective Study of Temporomandibular Joint Internal Derangement Treated with Arthrocentesis and Arthroscopy. Proceedings of Singapore Healthcare 2012;21:73-8.
30. Gonzalez-Garcia R, Rodriguez-Campo FJ, EscorialHernandez V, Munoz-Guerra MF, Sastre-Perez J, Naval-Gias L, et al. Complications of temporomandibular joint arthroscopy: a retrospective analytic study of 670 arthroscopic procedures. J Oral Maxillofac Surg 2006;64:1587-91.

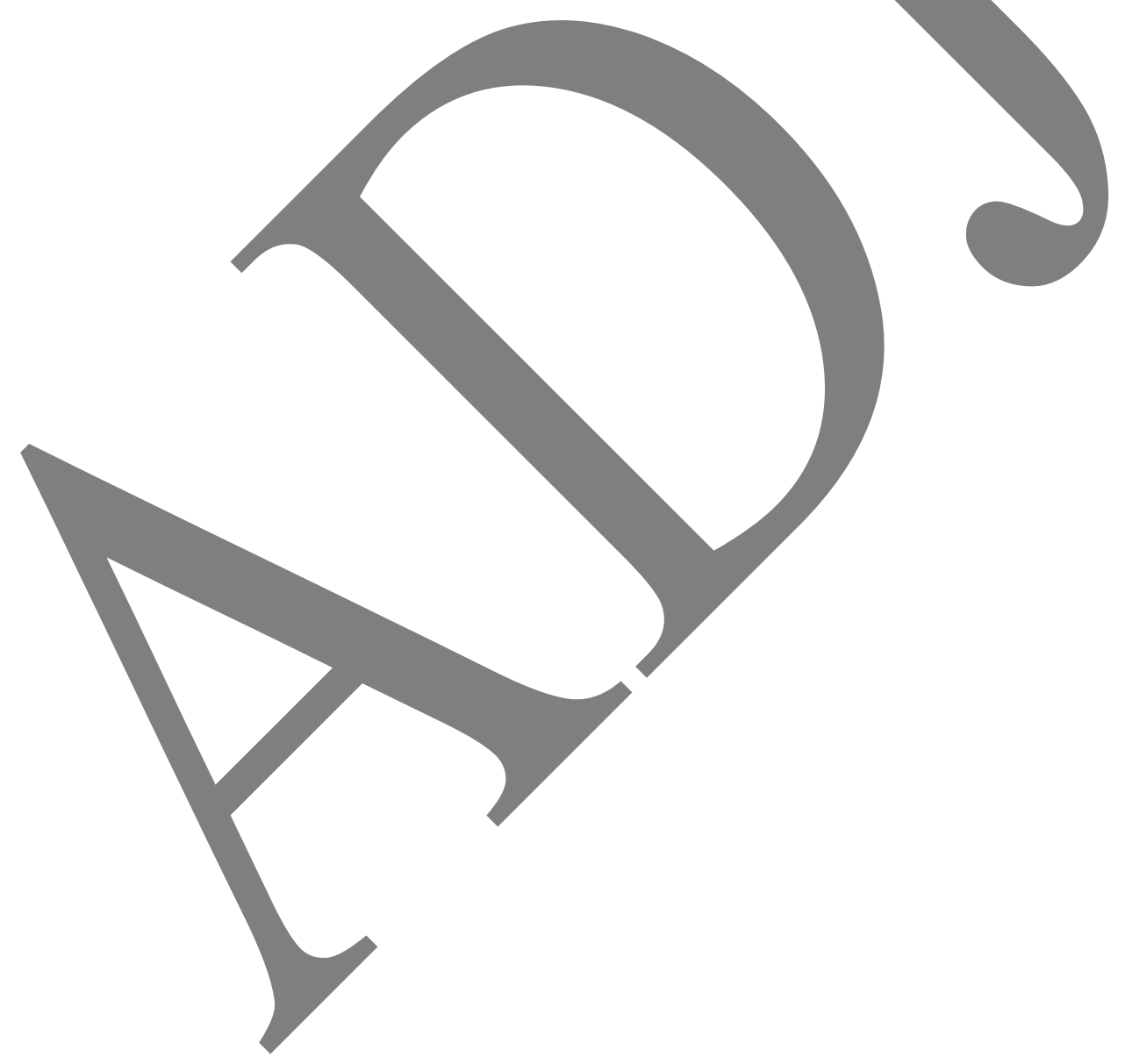

